SLAC-TN-10-036

LCLS-TN-08-8

\title{
Undulator Changes Due To Temperature Excursions
}

\author{
Zachary Wolf, Yurii Levashov, Ed Reese \\ SLAC
}

September 3, 2008

\begin{abstract}
The temperature of the LCLS undulators has not been controlled during storage. The effects of the temperature excursions are documented in this note.
\end{abstract}

\section{Introduction $^{1}$}

After a number of LCLS undulators were tuned, fiducialized, and placed in storage anticipating their use, a test was made to ensure that their properties had not changed. The test revealed, however, that indeed the undulators had changed. Detailed study of this problem followed. We now believe that the gap of the undulators changes permanently when the undulators go through temperature excursions. We have tested the other possible cause, transportation, and do not see gap changes. In this note, we document how the undulators have changed since they were originally tuned.

The undulators were tuned and fiducialized in the Magnetic Measurement Facility (MMF). Afterward, many of them (approximately 18) were taken to building 750 for storage during summer and fall 2007. Building 750 had no temperature control. The undulator temperatures went from $20 \mathrm{C}$, used for tuning, down to approximately $11 \mathrm{C}$ during the winter. In January 2008, three of the undulators were brought back to the MMF for a check. All three undulators showed similar changes. Trajectories, phases, and most undulator properties stayed the same, but the fiducialization (beam axis position relative to tooling balls on the undulator) had changed. Further investigation showed that the undulator gap was altered in a periodic way along the magnetic axis with a net average gap change causing the fiducialization change. A new storage location in building 33 was found and future undulators were placed there. A failure in the temperature control, however, caused the undulators to get too hot. Again the gap changed, but with a different periodic pattern. This note documents the measured changes in the undulators. In particular, it shows the detailed history of undulator 39 which went through both negative and positive temperature excursions.

\section{Undulator Changes}

\subsection{Undulator Gap Changes}

The poles of the undulators are canted, requiring care in measuring the gap. Each pole face was probed with the CMM to determine the vertical (y) position at six horizontal (x) positions. A linear fit was made to determine the $\mathrm{y}$ position of the pole face as a function of $\mathrm{x}$. A fixed $\mathrm{x}$ position relative to the tooling balls on the undulator was used in the fits to compare gaps. The fitted y positions of the upper and lower poles at the given $\mathrm{x}$ position were subtracted to determine the gap size. The following subsections show gap changes at a fixed $\mathrm{x}$ position.

\footnotetext{
${ }^{1}$ Work supported in part by the DOE Contract DE-AC02-76SF00515. This work was performed in support of the LCLS project at SLAC.
} 
The error on a gap measurement comes from two sources. The first is the uncertainty in the $\mathrm{x}$ position at which the gap is determined relative to tooling balls on the undulator. We estimate the uncertainty in the $\mathrm{x}$ position to be on the order of $10 \mu \mathrm{m}$. This error comes from locating the tooling balls and determining the coordinate system in which to measure $\mathrm{x}$. The resulting uncertainty in the gap, however, is $10 \mu \mathrm{m}$ times the $4.5 \mathrm{mrad}$ cant angle, which is negligible.

The primary error comes from the position measurements of points on the pole faces. To estimate the resulting error on the gap, we refer to a CMM calibration procedure in which the surface of a precision $3 \mathrm{~cm}$ diameter sphere was measured at 25 points. The measured points were fit to determine the center of the sphere. The maximum deviation of the measured radius from the true radius was determined to be $0.7 \mu \mathrm{m}$. We use this as our estimate of the CMM accuracy for measuring the gap of a single pole pair. It is an estimate of the CMM's accuracy for measuring the distance between nearby points.

The average gap of the undulator is calculated by averaging the gap measurements of all the poles. There are 226 pole pairs and therefore the statistical part of the error on the average gap is small. The error on the average gap, which comes from the systematic part of the single gap measurement error, is expected to be smaller than $0.7 \mu \mathrm{m}$.

\subsubsection{T $<<20$ C Gap Changes}

Three undulators with serial numbers 20,37, and 39 were brought back to the MMF from building 750 after they were stored there since the beginning of the winter. The measured undulator gap changed in the same manner for all three undulators. The gap changes and temperature histories of the three undulators are shown in figure 1. More detailed plots will follow, but here we stress the general nature of our conclusions. We will now concentrate on undulator 39 since it has the most extensive set of measurements.

Undulator 39 was first tuned on 10/31/07. The results are in dataset 1 . The datasets are referenced in the figures. Undulator 39 was placed in storage in building 750 . The building provided cover for the undulators, but no temperature control. Figure 2 shows the temperature history of undulator 39 from the time it left the MMF until it was returned. The undulator was tuned at $20 \mathrm{C}$ and went down to around $11 \mathrm{C}$ at the coldest point. The undulator was returned to the MMF and re-measured on 1/15/08 at $20 \mathrm{C}$. The results are in dataset 2. Figure 3 shows the change in undulator gap during the storage, i.e. the measured gap from dataset 2 minus the gap from dataset 1 . The size of the gap change is roughly $\pm 4 \mu \mathrm{m}$, which exceeds the estimated measurement error. Note the periodic structure in the gap difference. The pole assemblies are made up of sub-assemblies. The vertical lines in figure 3 show the boundaries of the sub-assemblies. The periodic structure in the plot is related to the length of the sub-assemblies. Note also that the average gap changed by $-0.7 \mu \mathrm{m}$, it became smaller. This is close to the accuracy of the CMM, but it is confirmed by the change in the fiducialization. We will come back to this when we discuss fiducialization changes. The measured magnetic field changes had the same periodic structure as the gap changes, but the magnetic field was larger where the measured gap was smaller and vice versa. We will come back to magnetic changes in a later section.

\subsection{2 $T=20 \pm 2$ C Gap Changes}

After taking dataset 2, undulator 39 stayed in a storage area of the MMF until a temperature controlled storage location was found. The undulator was then re-measured, and re-fiducialized on $4 / 9 / 08$. The results are in dataset 3. The difference between dataset 3 and dataset 2 shows the changes which occurred in the MMF storage area. (The MMF storage area is different than the tuning laboratory. The storage area provides only moderate temperature control.) The temperature of the MMF storage area during this time is shown in figure 4. The change in gap is shown in figure 5. Note that a periodic structure is evident again. The magnitude of the periodic gap change is 
smaller than when the undulator had the large temperature excursion. The average gap became larger by $0.6 \mu \mathrm{m}$ relative to dataset 2 .

\subsubsection{T $>>20$ C Gap Changes}

Temperature controlled storage was obtained in building 33. After dataset 3, undulator 39 was placed there. On 4/11/08, however, the temperature control system broke and the undulator temperature rose above $20 \mathrm{C}$. Figure 6 shows the room temperature recorded in the building 33 storage area. The temperature rose approximately $7 \mathrm{C}$. The temperature rise in the undulator crates was smaller, however. Figure 7 shows the temperature in the undulator 22 storage crate. (The undulator 39 temperature was not recorded.) The temperature rise in the storage crate was only about $3 \mathrm{C}$. Undulator 39 was brought back to the MMF, and on 6/10/08 dataset 4 was taken. The difference between dataset 4 and dataset 3 is shown in figure 8 . Note that the pattern of gap change is different in this case where the undulator got too hot than in the building 750 case where it got too cold. The average gap became larger by $0.7 \mu \mathrm{m}$ relative to dataset 3 .

\subsection{4 $T=20 \pm 0.1 \mathrm{C}$ Gap Changes}

After dataset 4, undulator 39 remained in the tightly temperature controlled tuning laboratory of the MMF awaiting reliable storage. On 6/19/08, we took a supposed final data set, dataset 5 . During the time from $6 / 10 / 08$ to $6 / 19 / 08$, the undulator remained at $20 \pm 0.1 \mathrm{C}$. The difference in gap measurements from dataset 4 to dataset 5 is shown in figure 9 . The pattern is absent. There is, however, a $-0.6 \mu \mathrm{m}$ average gap change, which is not confirmed by magnetic measurements and fiducialization changes.

\subsubsection{Summary Of Undulator Gap Changes}

A plot of all the gap measurements relative to those of dataset 1 are shown in figure 10. This plot clearly shows that the gap change when the undulator is heated is different than when the undulator is cooled. The pattern of change is different. It also shows that the process was not corrected by changing the temperature in the other direction.

\subsection{Undulator Fiducialization Changes}

An undulator is fiducialized by giving the position of the beam axis, a line along which $K$ has a specified value, relative to the tooling balls on the undulator. The $K$ value of the undulator depends on the magnetic field strength, which depends on the gap. The undulator poles have a nominal cant angle of $4.5 \mathrm{mrad}$, making the undulator gap depend on the $\mathrm{x}$ position. If the gap changes due to external factors such as thermal expansion distortions, a new x position is required to get to the original $K$ value, or equivalently, to the original gap. In this section we document the beam axis position changes for the various datasets of undulator 39, and also undulators 20 and 37 from building 750, and undulator 16 from building 33 .

The table below shows the change in the undulator 39 beam axis position for each dataset. The axis position is given relative to fixed tooling balls on the undulator. The numbers in the table show the changes in axis position both relative to the previous dataset and also relative to the first dataset. Axis shifts in both $\mathrm{x}$ and $\mathrm{y}$ are given. Note that the axis y position is very stable. The $\mathrm{x}$ position changes to compensate for average gap changes.

We estimate the accuracy of the fiducialization process by using data from the reference undulator. The reference undulator is kept permanently in the MMF tuning laboratory at $20 \pm 0.1 \mathrm{C}$ and is measured and fiducialized approximately once per month. The reference undulator fiducialization data provides a worst case error estimate since changes in the reference undulator are included in this estimate. Laboratory temperature fluctuation effects are also included. The measured beam 
axis of the reference undulator shifted in $\mathrm{x}$ by approximately $50 \mu \mathrm{m}$ during the $2 \frac{1}{2}$ months between undulator 39 datasets 1 and 2. Shifts in the reference undulator fiducialization between the other datasets of undulator 39 were comparable or smaller. We therefore use $50 \mu \mathrm{m}$ as the estimated accuracy of the numbers in the first row of the table. We will use this estimate below when we calculate the gap change corresponding to the fiducialization change.

The average gap changes measured by the CMM are included at the bottom of the table. The average gap change values are comparable to the accuracy of the measurement, which we estimated to be smaller than $0.7 \mu \mathrm{m}$.

We also calculate the average gap change relative to the previous dataset by using the beam axis $\mathrm{x}$ position changes given in the first line of the table. The calculated average gap change is given by the $\mathrm{x}$ position change times the cant angle. The cant angle is measured by the CMM for every pole pair. The average cant angle for undulator 39 is $4.8 \mathrm{mrad}$. The bottom line of the table shows the calculated gap change relative to the previous dataset. The error on the calculated gap change is $50 \mu \mathrm{m}$ times $4.8 \mathrm{mrad}$, or $0.2 \mu \mathrm{m}$. Within errors, there is agreement between the gap change measured by the CMM and the gap change determined by the fiducialization shifts in $\mathrm{x}$.

From dataset 1 to dataset 2, the fiducialized beam axis shifted in $\mathrm{x}$ by $-197 \mu \mathrm{m}$. This corresponds to the axis shifting in the direction of larger gap in the canted poles because the temperature effect made the average gap smaller. The $-197 \mu \mathrm{m}$ shift with a cant angle of $4.8 \mathrm{mrad}$ gives a calculated gap change of $-0.9 \mu \mathrm{m}$, which is consistent with CMM measurement of $-0.7 \mu \mathrm{m}$.

The change in the $\mathrm{x}$ axis position between dataset 3 and dataset 2 is $145 \mu \mathrm{m}$. This corresponds to a calculated gap change of $0.7 \mu \mathrm{m}$. This is consistent with the CMM measurement of $0.6 \mu \mathrm{m}$.

The axis shift between dataset 4 and dataset 3 is $132 \mu \mathrm{m}$. This corresponds to a calculated gap change of $0.6 \mu \mathrm{m}$. This is consistent with the CMM measurement of $0.7 \mu \mathrm{m}$.

The axis shift between dataset 5 and dataset 4 is $-63 \mu \mathrm{m}$. The CMM measurements show an average gap change of $-0.6 \mu \mathrm{m}$. The calculated gap change is $-0.3 \mu \mathrm{m}$, however. The sign of the axis shift and gap change are consistent. The magnitudes, however, do not agree well, but are consistent within errors.

\begin{tabular}{|r|r|r|r|r|r|}
\hline Dataset & 1 & 2 & 3 & 4 & 5 \\
\hline X Axis wrt Previous $(\mu \mathrm{m})$ & 0 & -197 & 145 & 132 & -63 \\
\hline X Axis wrt Dataset 1 $(\mu \mathrm{m})$ & 0 & -197 & -53 & 79 & -17 \\
\hline Y Axis wrt Previous $(\mu \mathrm{m})$ & 0 & -8 & 1 & -9 & 12 \\
\hline Y Axis wrt Dataset 1 $(\mu \mathrm{m})$ & 0 & -8 & -6 & -15 & -3 \\
\hline \hline Gap wrt Previous $(\mu \mathrm{m})$ & 0 & -0.7 & 0.6 & 0.7 & -0.6 \\
\hline Calc Gap wrt Previous $(\mu \mathrm{m})$ & 0 & -0.9 & 0.7 & 0.6 & -0.3 \\
\hline
\end{tabular}

As noted above, undulators 20 and 37 were also re-measured after being stored in building 750 . Undulator 20 was re-fiducialized on $1 / 3 / 08$. The beam axis shifted in $\mathrm{x}$ by $-134 \mu \mathrm{m}$, and in $\mathrm{y}$ by $-8 \mu \mathrm{m}$. Undulator 37 was re-fiducialized on $1 / 15 / 08$. The beam axis shifted in $\mathrm{x}$ by $-201 \mu \mathrm{m}$, and in $\mathrm{y}$ by $-15 \mu \mathrm{m}$. These values agree with the fiducialization changes in undulator 39 between dataset 1 and dataset 2. We conclude that undulators 20,37, and 39 had similar fiducialization changes when subjected to the cold temperatures.

Undulator 16 was re-measured after being stored in building 33. Dataset 2 re-fiducialization data was taken on $7 / 3 / 08$. The beam axis shifted in $\mathrm{x}$ by $130 \mu \mathrm{m}$, and in y by $8 \mu \mathrm{m}$. This agrees with the fiducialization changes in undulator 39 between dataset 3 and dataset 4 .

\subsection{Undulator Magnetic Changes}

The change in the measured magnetic pole tip fields relative to the first dataset are shown in figure 11. The field changes correspond to the gap changes measured by the CMM. Note that the magnetic measurements are done on the magnetic axis where $\mathrm{K}$ is constant. Average field changes are not seen in this plot. The pattern, however, confirms the pattern seen in the gap changes. 
The field changes do not significantly affect the trajectories. Figure 12 shows the x-trajectory for each of the five datasets. Figure 13 shows the y-trajectories. All trajectories stay within tolerance.

The field changes do cause some extra phase errors. The phase relative to the first dataset is shown in figure 14. The extra phase errors are small, however, and are well within tolerance.

We conclude that the primary magnetic effect of undulator temperature excursions is a $K$ shift on the fiducialized beam axis. The shift is due to the average undulator gap changing which causes average magnetic field strength changes. The periodic pattern of gap changes does not cause significant trajectory or phase errors.

\section{Conclusion}

The LCLS undulators are sensitive to temperature. Even two degree Celsius changes permanently alter the undulator gap. Great care must be used when transporting, storing, and using the undulators.

\section{List Of Figures}

1. Undulators 20, 37, and 39 were re-measured after being stored in building 750 . All showed similar gap changes. The temperature histories are also shown.

2. Undulator 39 temperature history in building 750 .

3. Undulator 39 gap difference from before and after building 750 storage.

4. Temperature in the MMF storage area while undulator 39 was stored there.

5. Undulator 39 gap change from before and after MMF storage.

6. Ambient temperature in the building 33 storage area.

7. Temperature in the undulator 22 storage crate in building 33 .

8. Undulator 39 gap difference from before and after building 33 storage.

9. Undulator 39 gap difference from before and after MMF tuning laboratory storage.

10. Undulator 39 gap changes relative to dataset 1.

11. Undulator 39 peak field changes relative to dataset 1.

12. Undulator $39 \mathrm{x}$-trajectory for each dataset.

13. Undulator 39 y-trajectory for each dataset.

14. Undulator 39 phase relative to dataset 1. 


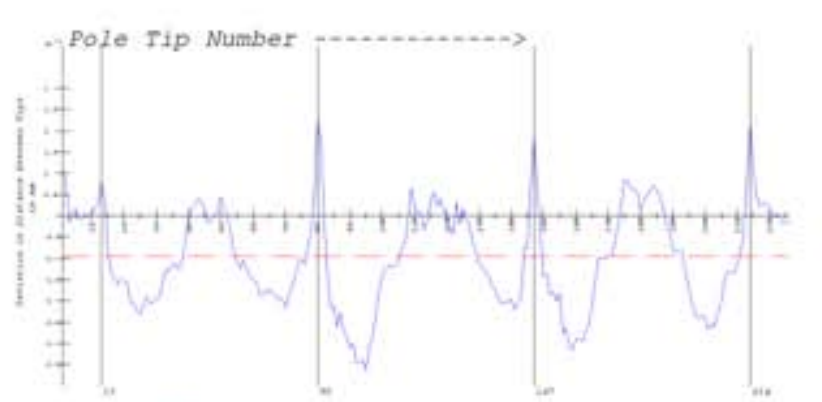

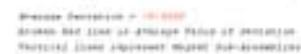

\begin{tabular}{|c|c|c|}
\hline 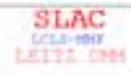 & 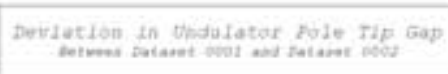 & 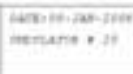 \\
\hline
\end{tabular}

\&

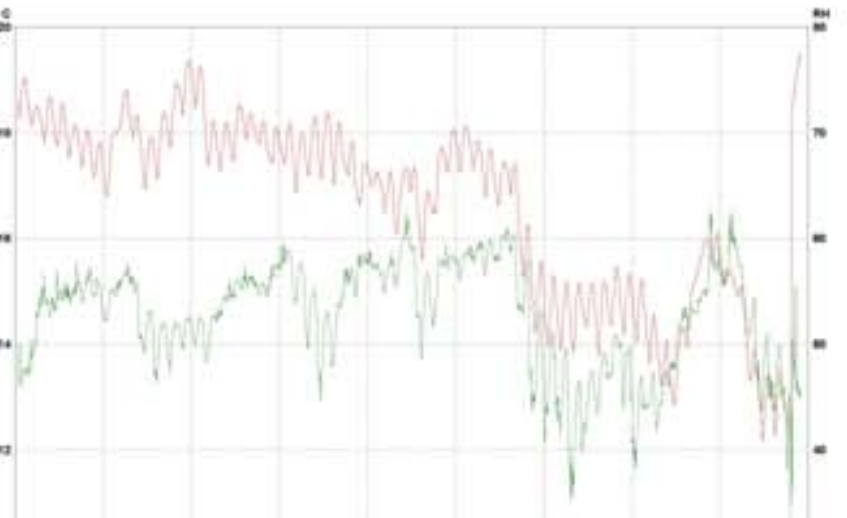

1

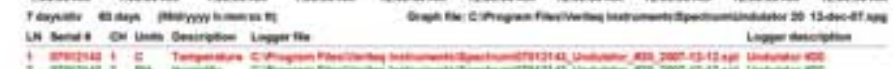

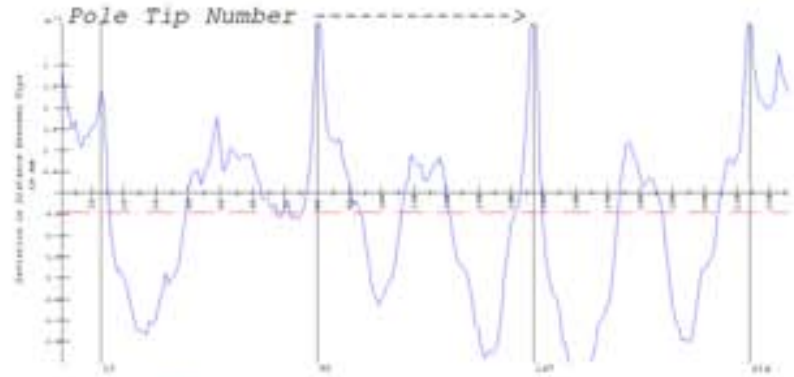

는

\begin{tabular}{|c|c|c|}
\hline 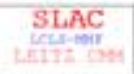 & 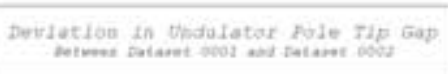 & 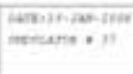 \\
\hline
\end{tabular}

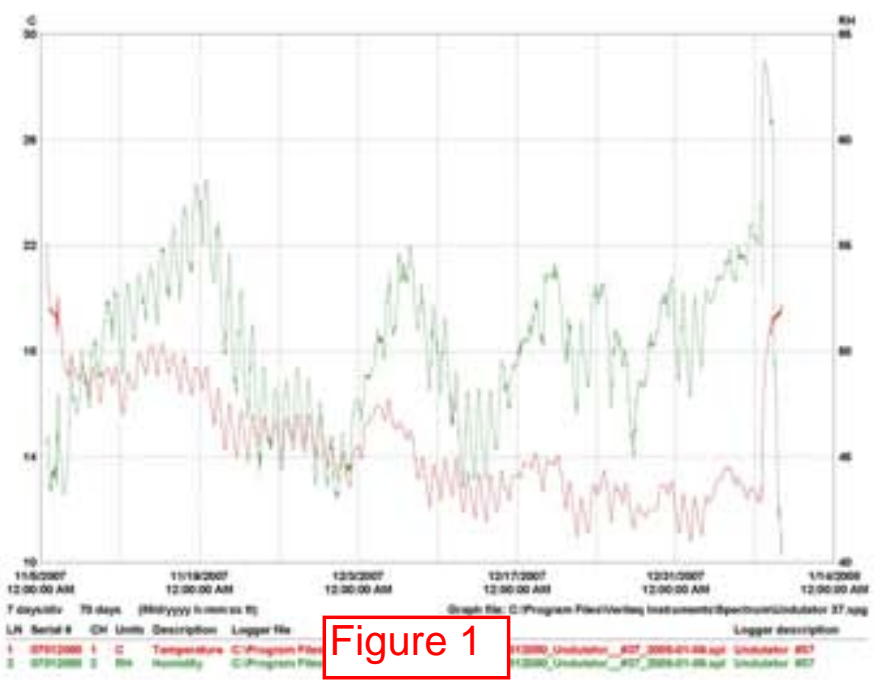

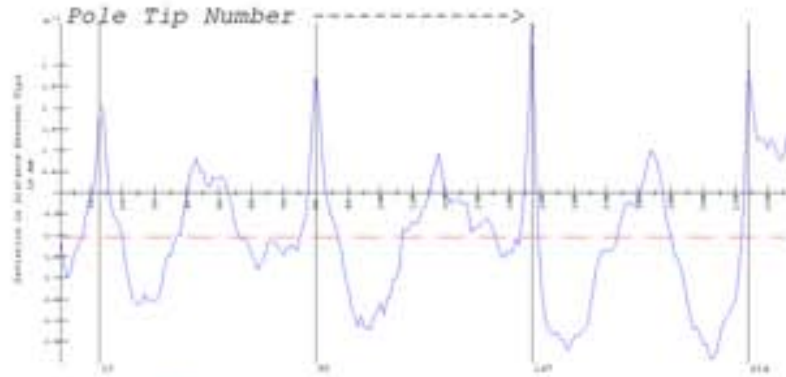

는

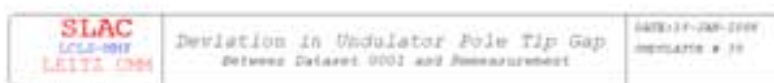

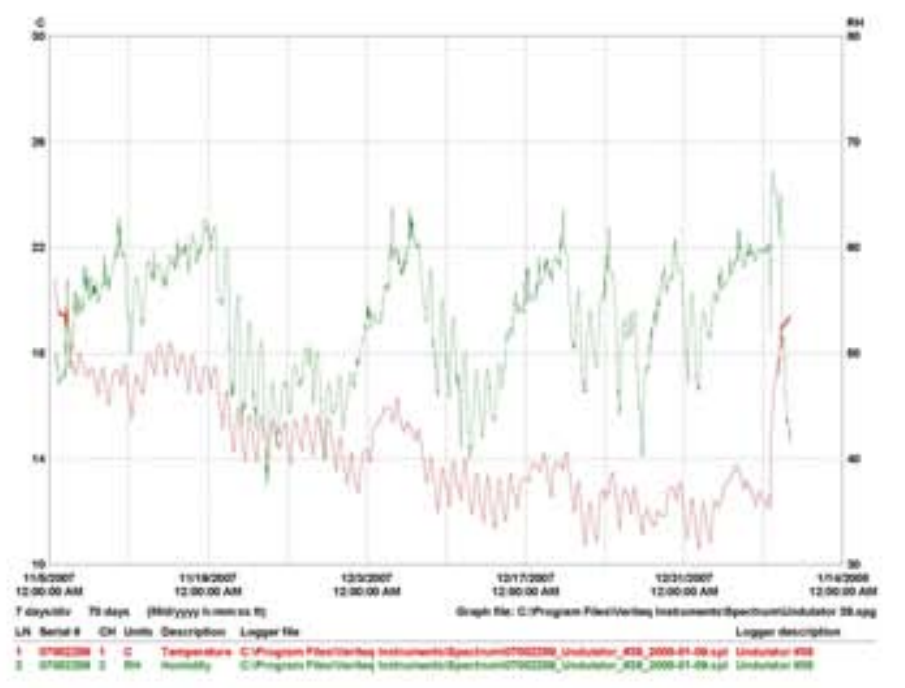


26

22

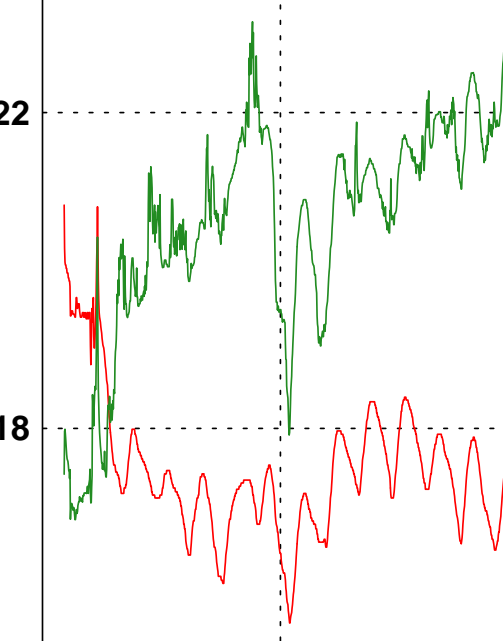

14
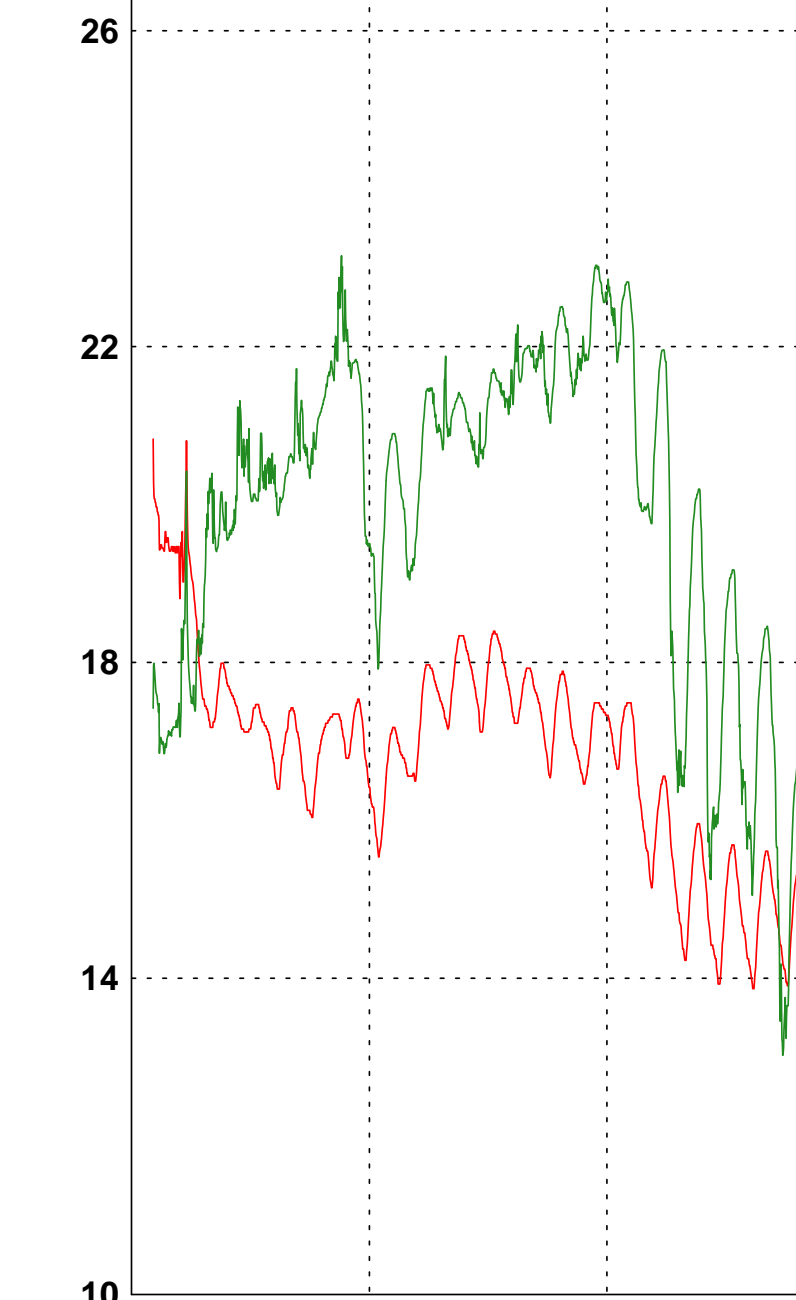

$$
10
$$

7 days/div 70 days (M/d/yyyy $h: m m: s s ~ t t)$

\section{LN Serial \# CH Units Description Logger file}

Graph file: C:|Program Files|Veriteq Instruments|Spectrum|Undulator 39.spg

$1070822891 \quad$ C $\quad$ Temperature C:IProgram Files|Veriteq Instruments ISpectrum 107082289 Undulator_\#39_2008-01-09.spl Undulator \#39

2070822892 RH Humidity C:IProgram Files $\backslash$ Veriteq Instruments $\backslash$ Spectrum $\backslash 07082289 \_U n d u l a t o r \_\# 39 \_2008-01-09 . s p l$ Undulator \#39 


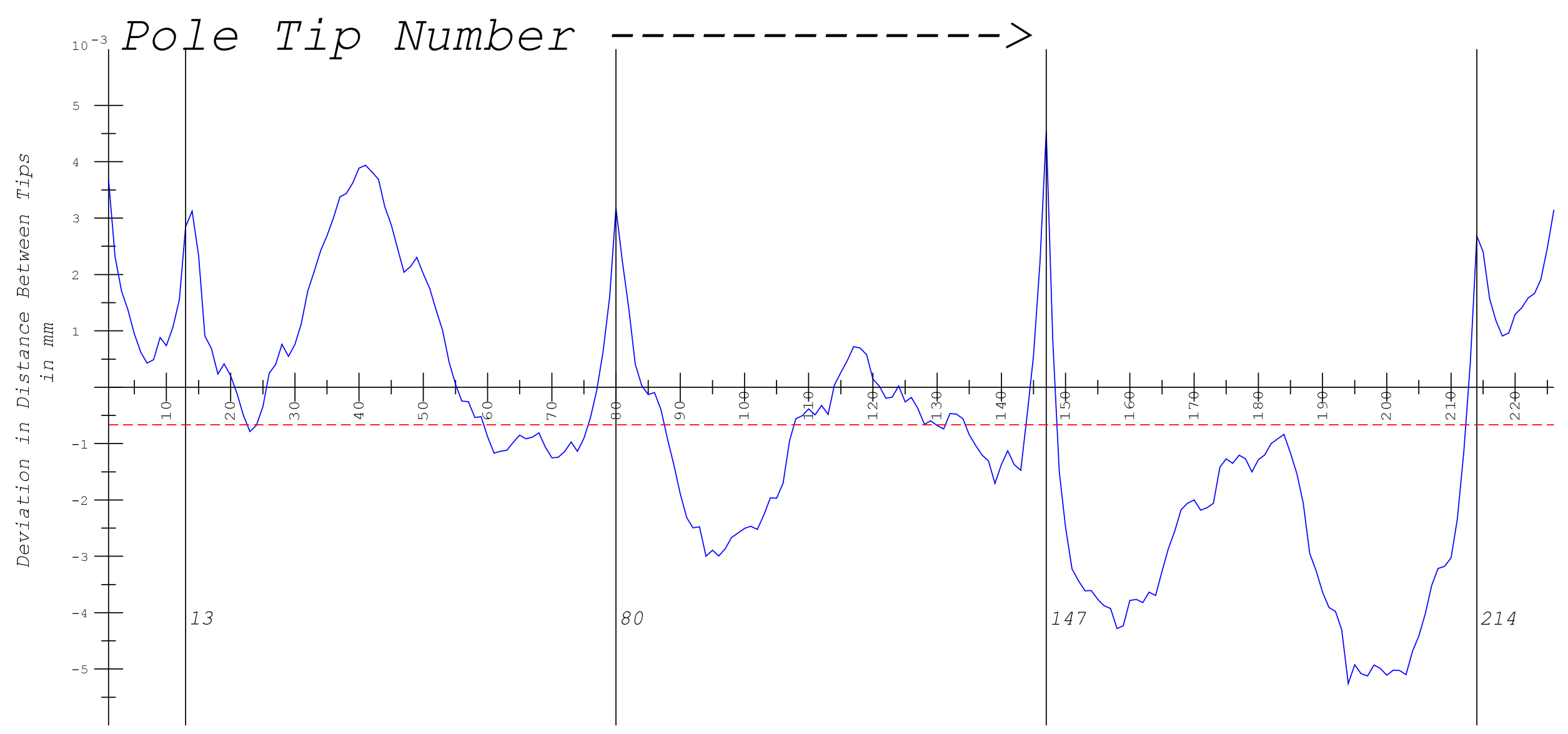

Average Deviation $=-0.0007$

Broken Red Iine is Average Value of Deviation

Vertical lines represent Magnet Sub-Assemblies

Deviation is the change in Gap width at the same yZ Cross section (Dataset oool Magnetic Centerline) New - Old

\section{SLAC} LCLS-MMF LEITZ CMM
Deviation in Undulator Pole Tip Gap Between Dataset 0001 and Dataset 0002
DATE:07-JUL-2008

UNDULATOR \# 39 


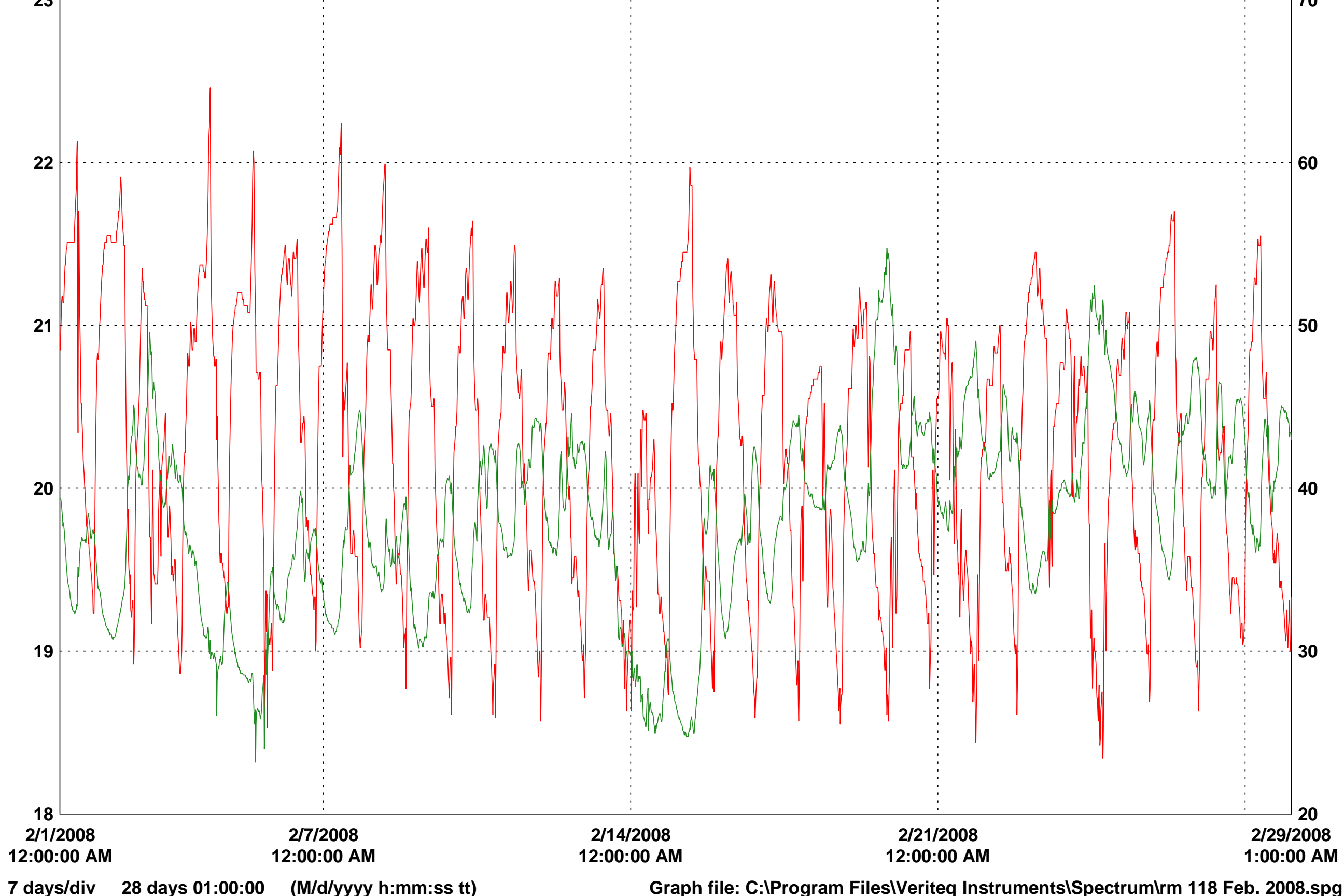

7 days/div 28 days 01:00:00 (M/d/yyyy $h: m m: s s ~ t t)$

Graph file: C:|Program Files|Veriteq Instruments|Spectrum $\backslash r m 118$ Feb. 2008.spg

LN Serial \# CH Units Description Logger file

Logger description

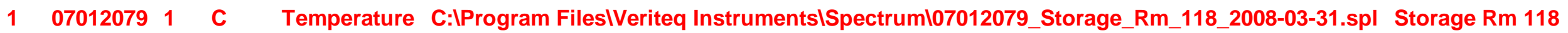

2070120792 RH Humidity C:IProgram FilesiVeriteq Instruments 1 Spectrum $\backslash 07012079$ Storage_Rm_118_2008-03-31.spl Storage Rm 118 Figure 4 


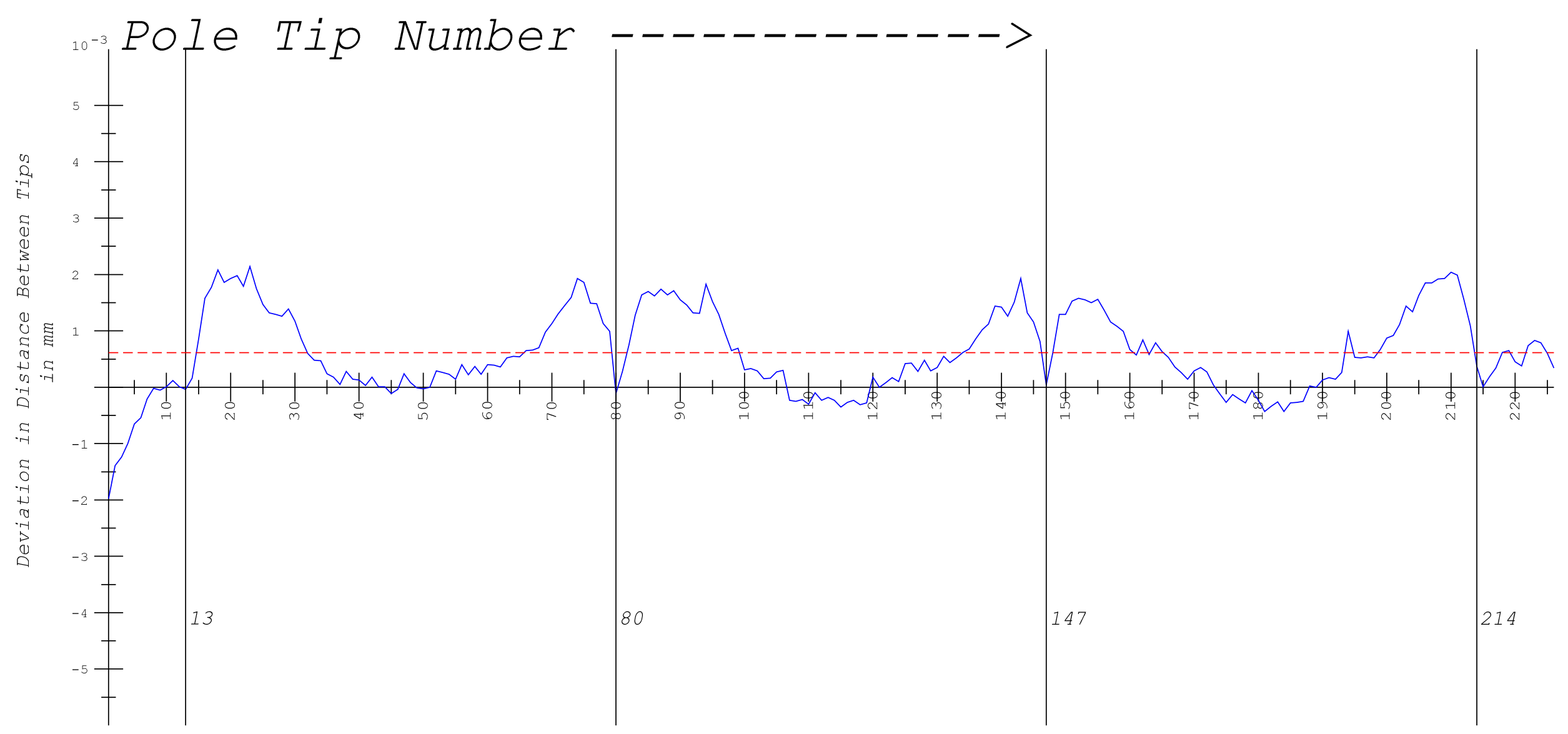

Average Deviation $=0.0006$

Broken Red line is Average Value of Deviation

Vertical lines represent Magnet Sub-Assemblies

Deviation is the change in Gap width at the same yZ Cross section (Dataset oool Magnetic Centerline) New - Old

\section{SLAC} LCLS-MMF LEITZ CMM
Deviation in Undulator Pole Tip Gap Between Dataset 0002 and Dataset 0003
$D A T E: 07-J U L-2008$

UNDULATOR \# 39 

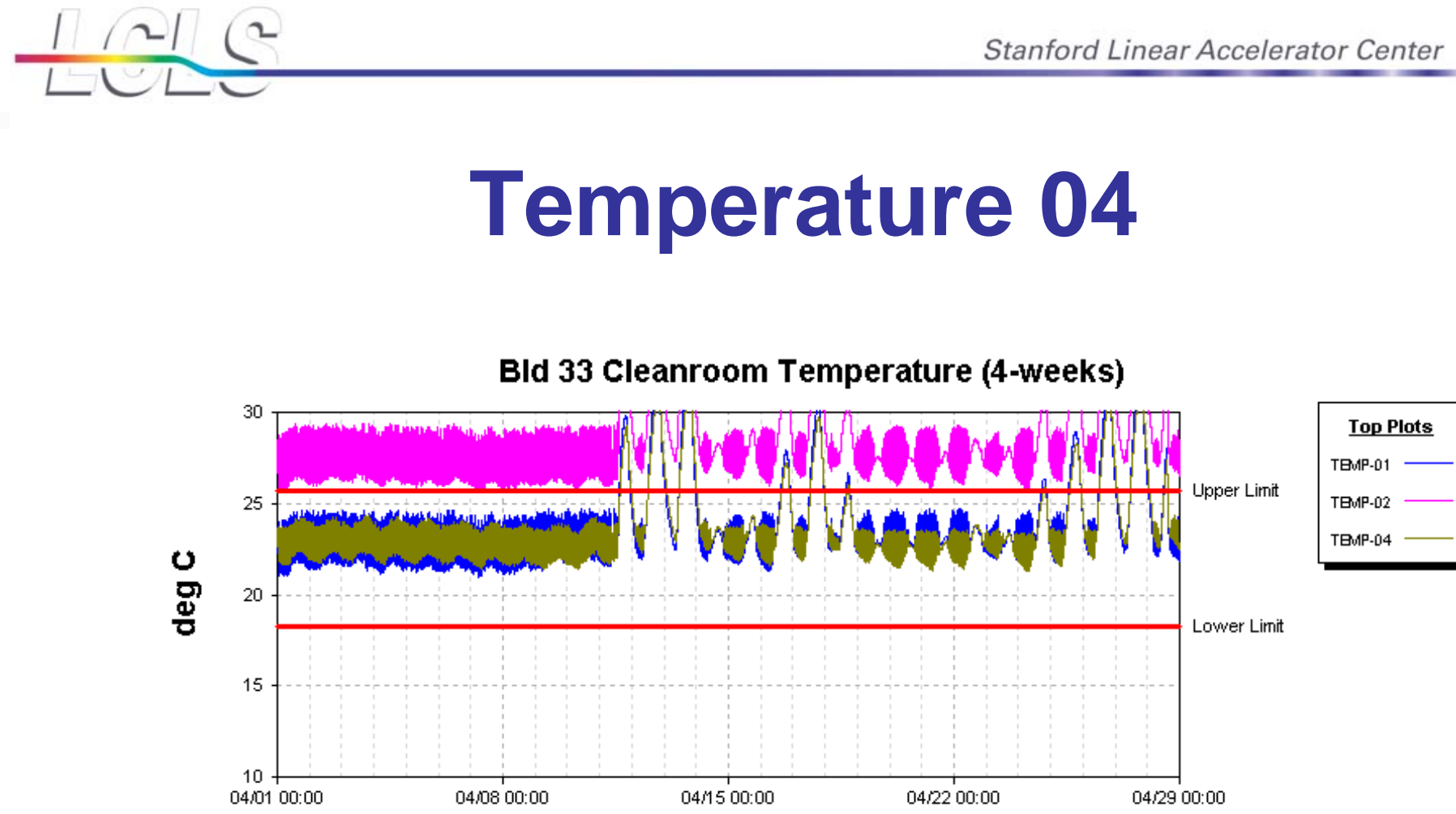

BId 33 Cleanroom Humidity (4-weeks)

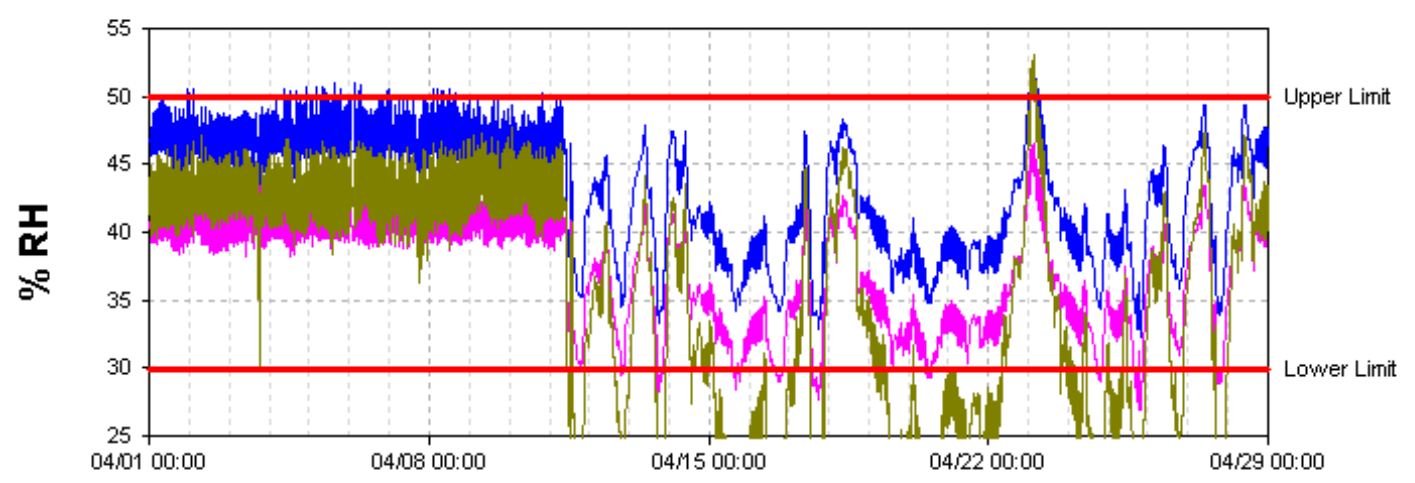

Bottom Plots

HUM-01

HUM-02

HuM-04

Time: 04/01/08 00:00:00 to 04/29/08 00:00:00, Duration: 28 days + 00:00:00

\section{4/29/08}

Undulator Week- Storage
Figure 6
Jose Chan

jqchan@slac.stanford.edu 


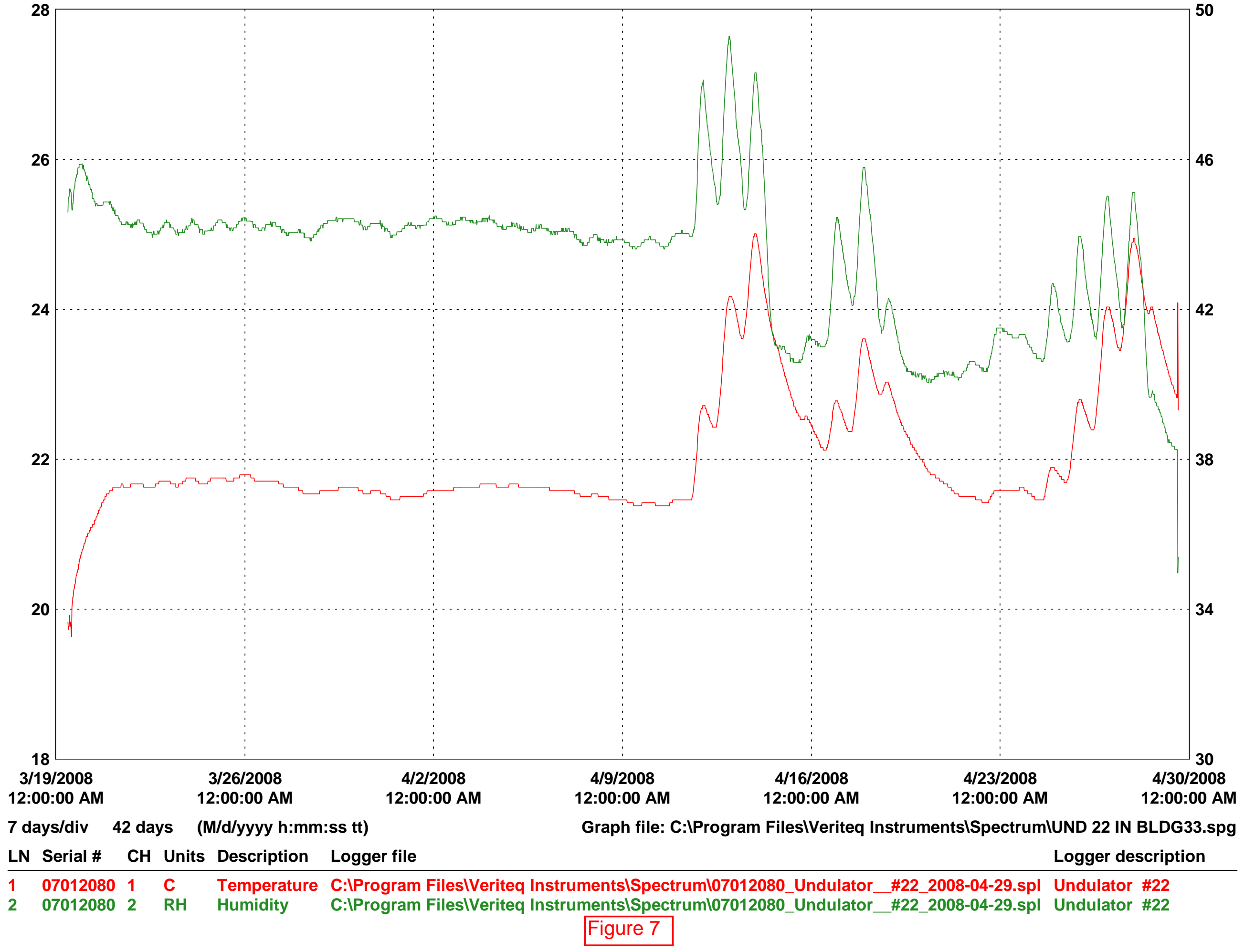




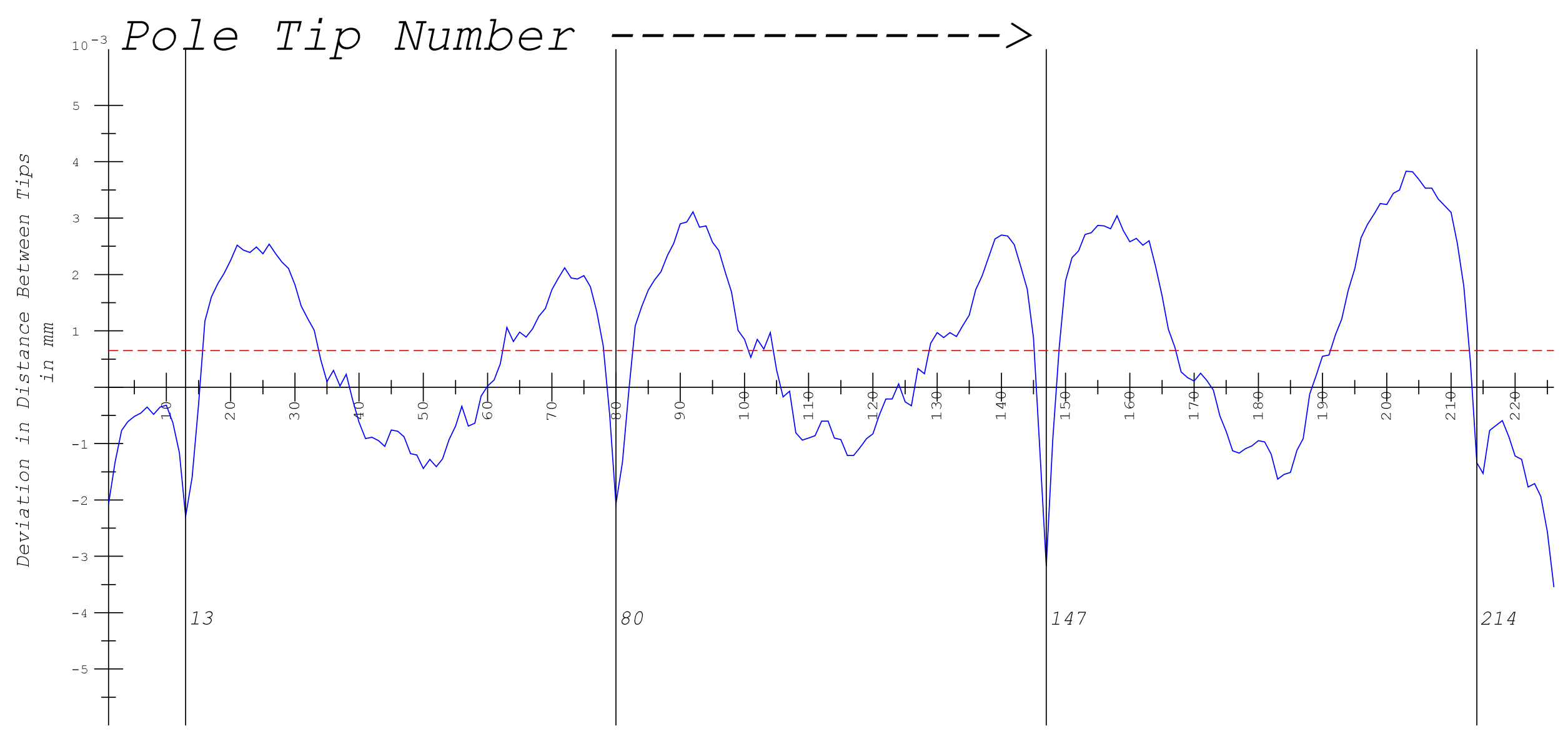

Average Deviation $=0.0007$

Broken Red Iine is Average Value of Deviation

Vertical lines represent Magnet Sub-Assemblies

Deviation is the change in Gap width at the same yZ Cross section (Dataset oool Magnetic Centerline) New - Old

\section{SLAC} LCLS-MMF LEITZ CMM
Deviation in Undulator Pole Tip Gap Between Dataset 0003 and Dataset 0004
$D A T E: 07-J U L-2008$

UNDULATOR \# 39 


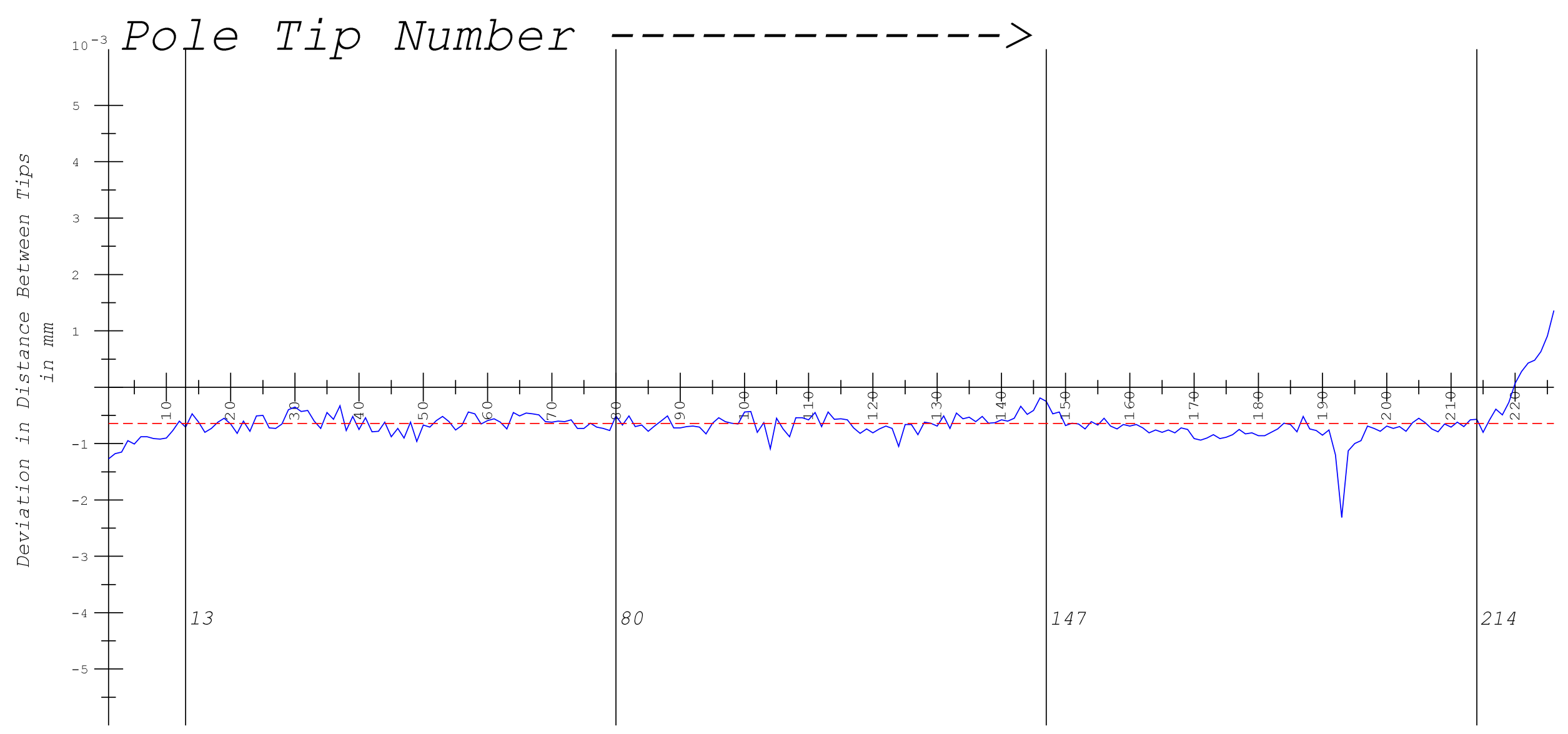

Average Deviation $=-0.0006$

Broken Red Iine is Average Value of Deviation

Vertical lines represent Magnet Sub-Assemblies

Deviation is the change in Gap width at the same yZ Cross section (Dataset oool Magnetic Centerline) New - Old

\section{SLAC} LCLS-MMF LEITZ CMM
Deviation in Undulator Pole Tip Gap Between Dataset 0004 and Dataset 0005
DATE:07-JUL-2008

UNDULATOR \# 39 


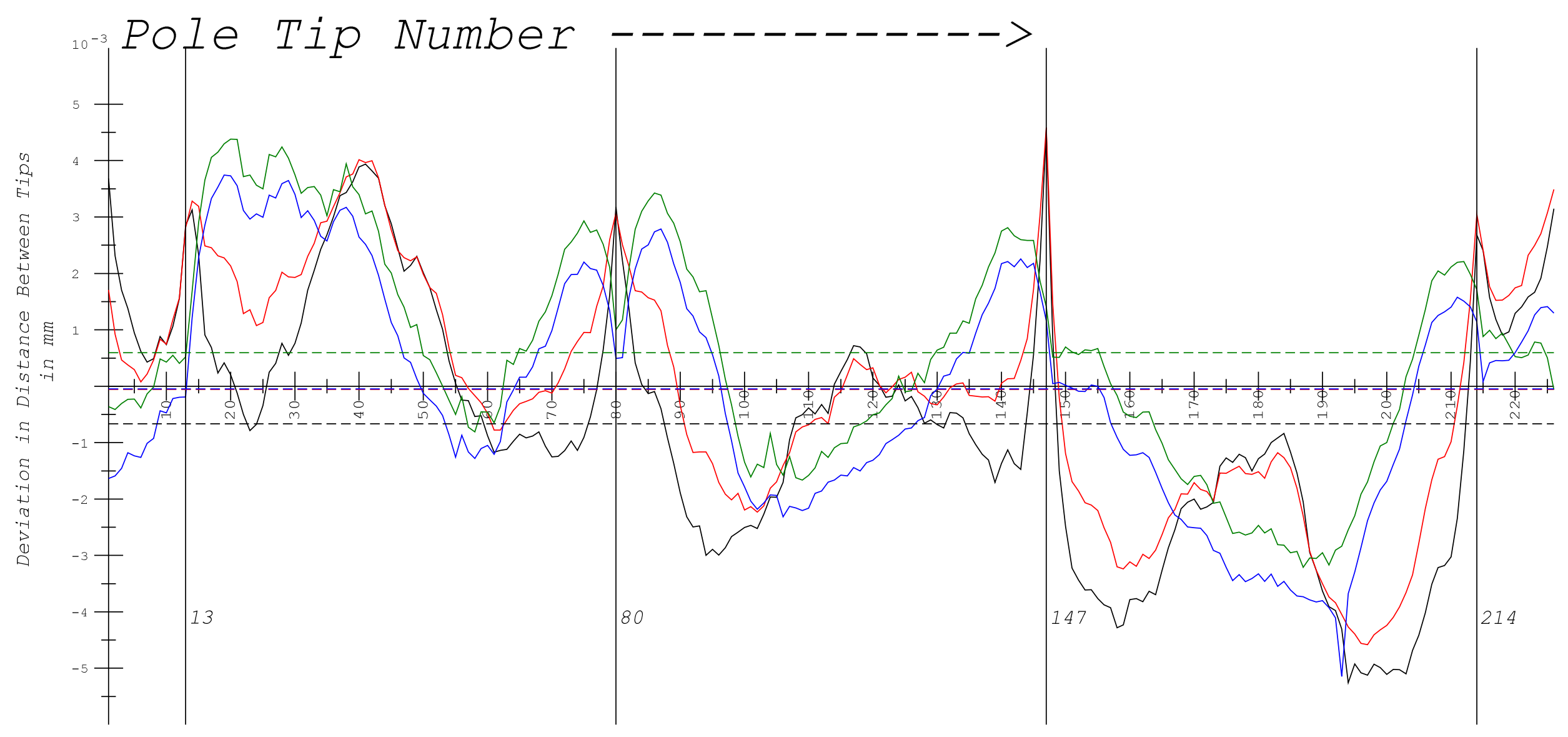

Average Deviation 0002-0001 $=-0.0007$ Average Deviation 0003-0001 $=-0.0001$

Average Deviation 0004-0001 $=0.0006$

Average Deviation 0005-0001 = 0.0000

Vertical lines represent Magnet Sub-Assemblies

Deviation is the change in Gap width at the same yz cross section (Dataset oool Magnetic Centerline) New - old

\section{SLAC} LCLS-MMF LEITZ CMM
Deviation in Undulator Pole Tip Gap Between Dataset 0002-5 and Dataset 0001
DATE:07-JUL-2008

UNDULATOR \# 39 


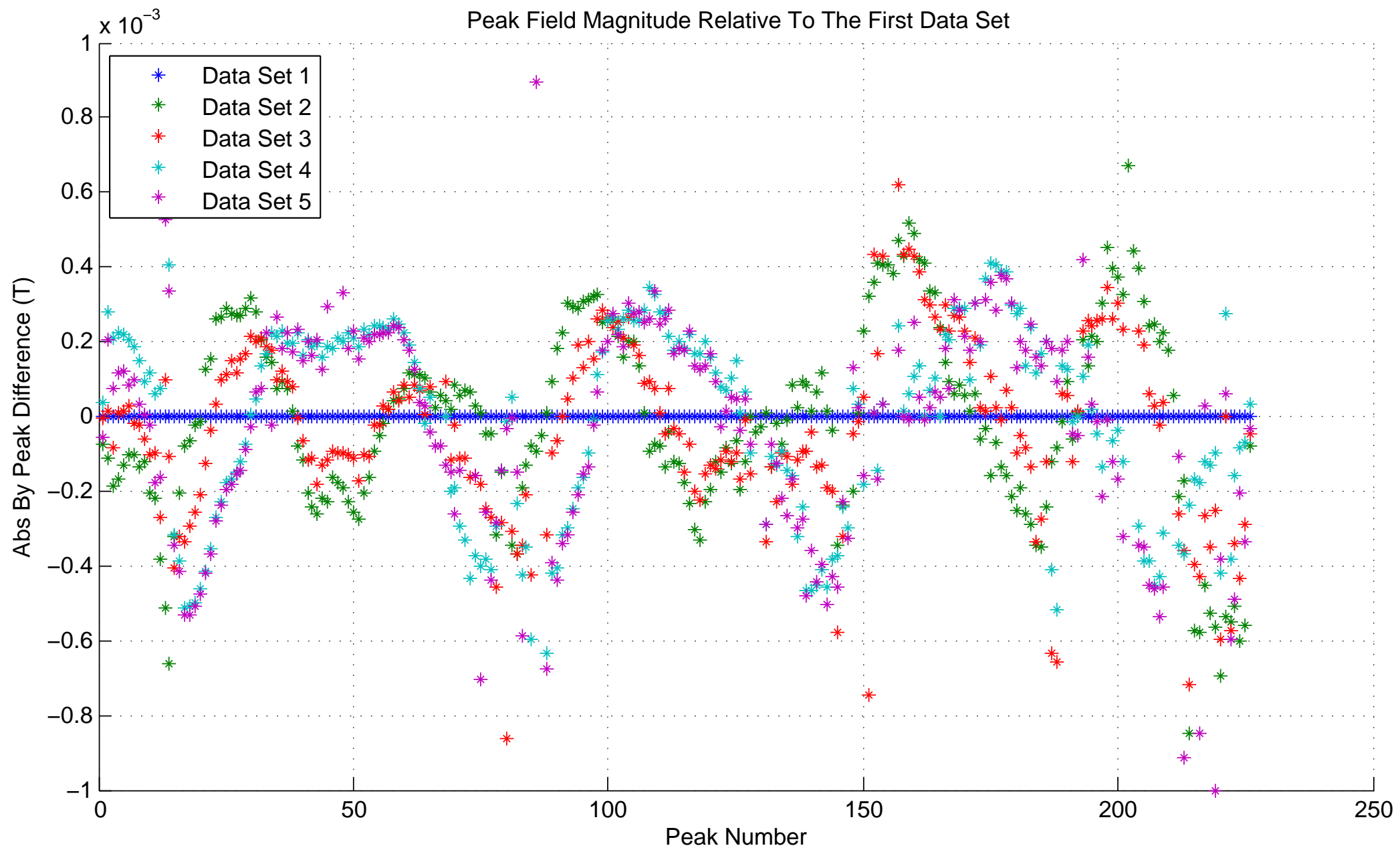

Figure 11 


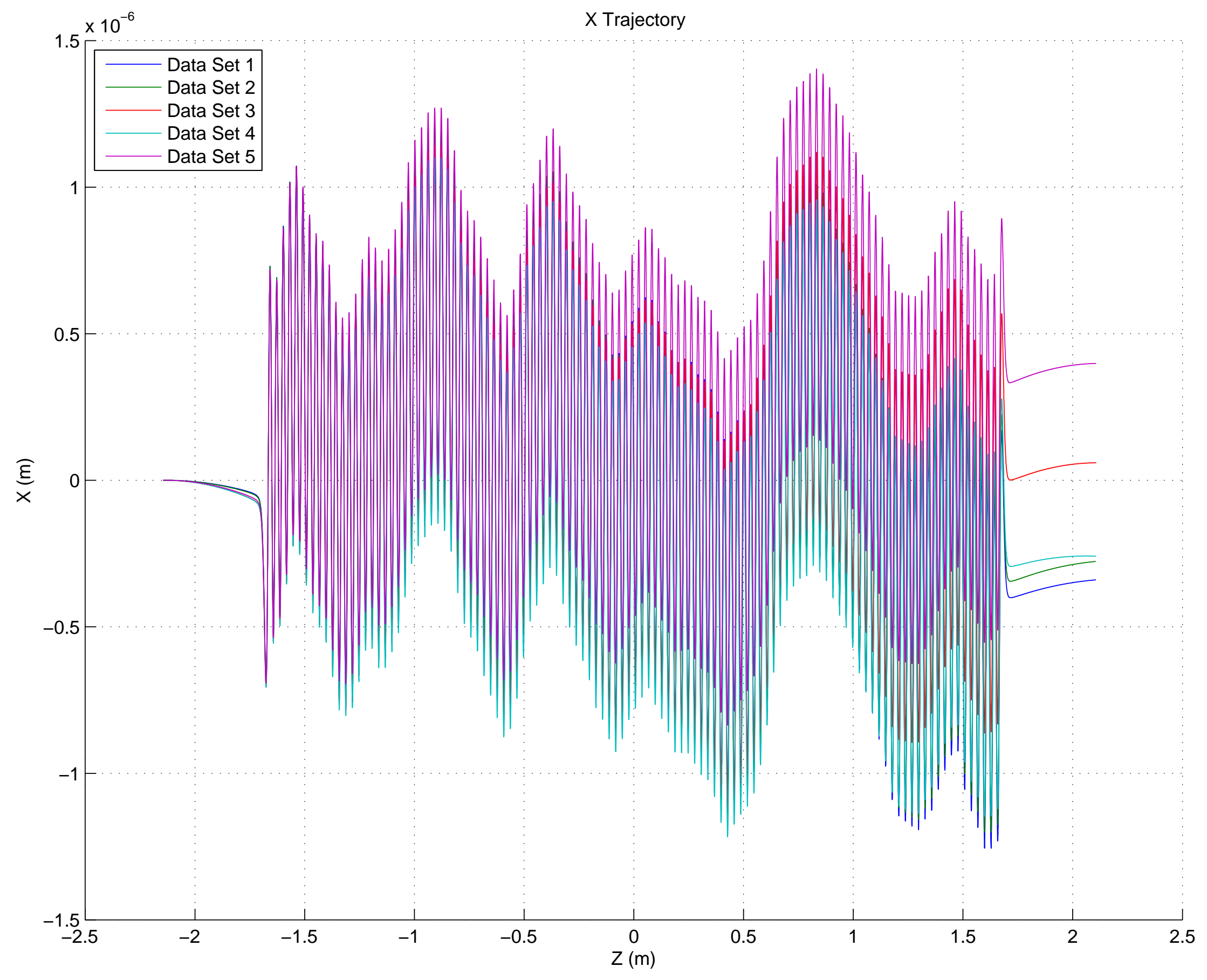

Figure 12 


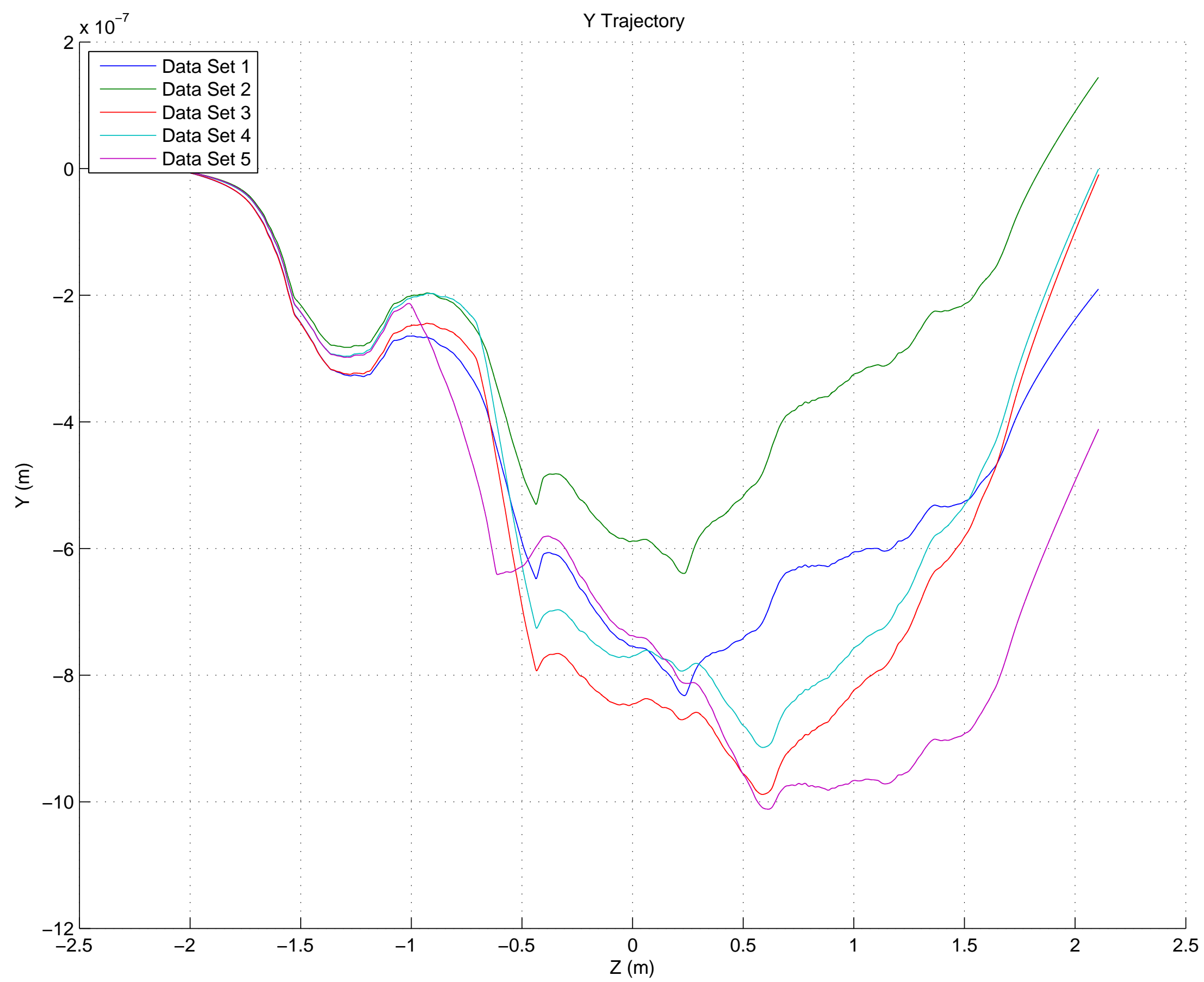

Figure 13 
Phase Relative To The Phase Of The First Data Set

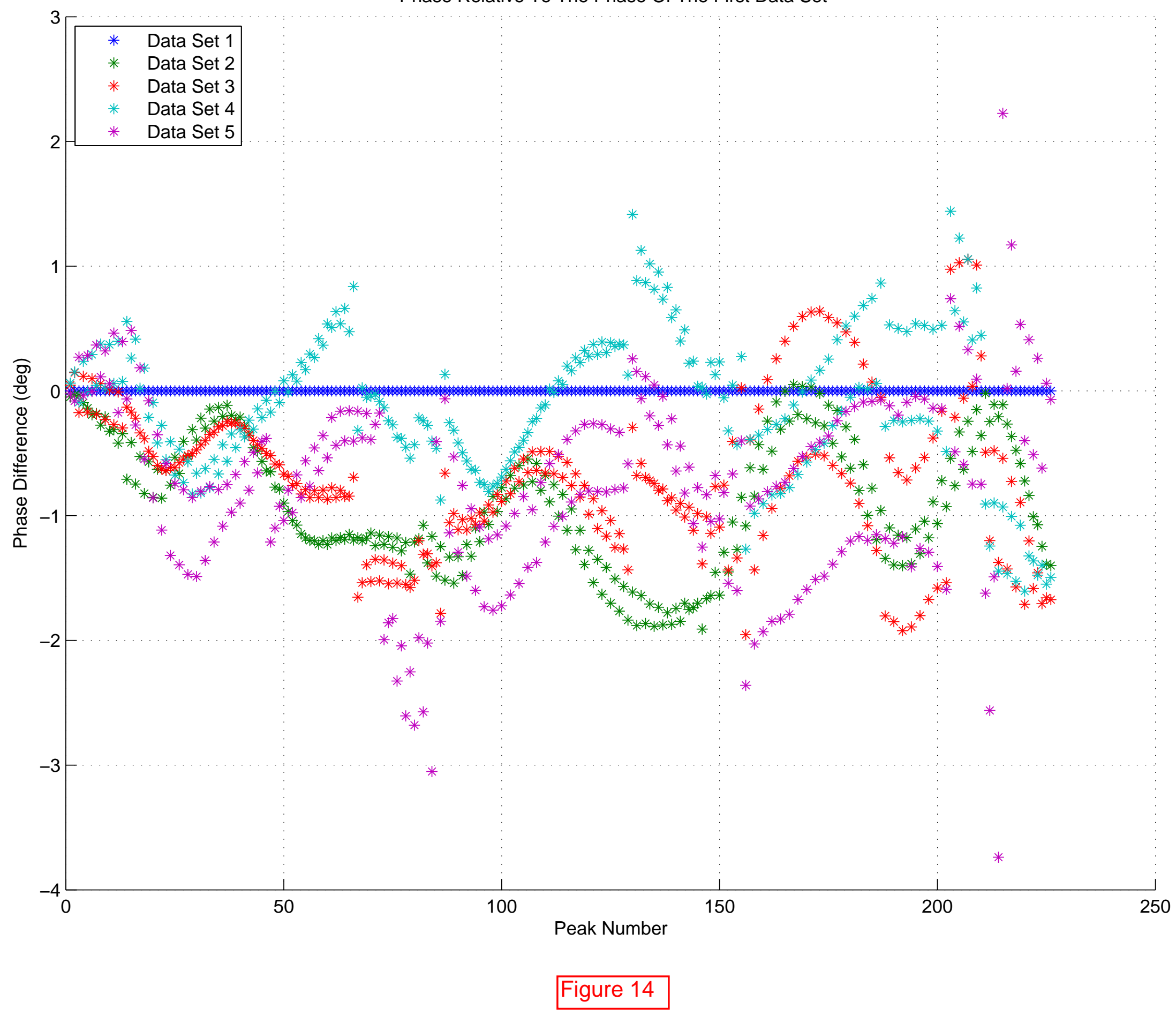

